\title{
CThE1.pdf
}

\section{Widely Tunable Yb:KYW Laser Locked by a Volume Bragg Grating}

\author{
Björn Jacobsson, Jonas E. Hellström, Valdas Pasiskevicius, Fredrik Laurell \\ Laser Physics, KTH - Royal Institute of Technology, 10691 Stockholm, Sweden \\ bj@laserphysics.kth.se
}

\begin{abstract}
We demonstrate a technique for laser tuning based on a volume Bragg grating. A peak power of $4.7 \mathrm{~W}$, a bandwidth $<0.1 \mathrm{~nm}$ and a 1000-1050 nm tuning range is achieved in an $\mathrm{Yb}: \mathrm{KYW}$ laser.

(C)2006 Optical Society of America

OCIS codes: (090.7730) Volume holographic gratings; (140.3600) Lasers, tunable
\end{abstract}

Wavelength tuning of solid-state lasers is typically obtained by use of intra-cavity elements which introduce losses at undesired wavelengths, such as etalons, birefringent filters and prisms. The performance of these elements depends on the wavelength selectivity, i.e. the introduced losses at wavelengths close to the desired wavelength. For many elements there is also a free spectral range with which the low loss of the desired wavelength repeats itself regularly, while other elements cause the need for cavity realignment while tuning. In this paper we introduce the use of an intra-cavity tuning element based on a volume Bragg grating. This element has an excellent wavelength selectivity without any free spectral range limitation and no need for cavity realignment.

Volume Bragg gratings consist of photosensitive glass with a sinusoidal refractive index modulation throughout the volume. These devices enable a high peak reflectivity combined with a very small reflection bandwidth on the sub-nanometer scale. Previously, volume Bragg gratings have been used to lock the wavelength of high-power diode lasers as well as various solid-state lasers [1]. However, in these cases the laser was locked to a single wavelength and could not be tuned more than permitted by temperature tuning of the Bragg peak, i.e. some nanometers at most.

Extensive tuning of the reflected wavelength from a Bragg grating can be achieved by turning the grating. The reflected wavelength, $\lambda$, is tuned with the (internal) angle of incidence $\theta$, according to $\lambda=\lambda_{0} \cos \theta$, where $\lambda_{0}$ is the wavelength at normal incidence. Naturally, turning the grating causes beam steering and thus requires repeated cavity realignment, which is very inconvenient if one wants to tune continuously. To avoid this problem, one possibility is to position the grating perpendicular to an ordinary broadband mirror. In this way, a retroreflector is obtained, which always reflects the beam backwards in the same direction, irrespective of the angle of incidence [2]. Now, by rotating the retroreflector around an axis at the crossing point of the two mirrors, the wavelength can be tuned without beam steering and the cavity alignment is preserved. Another problem is that when a finite beam is incident at an oblique angle onto a thick Bragg grating, the ordinary plane wave result for the reflectivity is no longer valid [3]. Due to the walk-off that occurs between the incident and reflected beams, the reflectivity is reduced with increased incidence angle and smaller beam radius. Therefore, it is important to have as large a beam size as possible on the Bragg grating.

For demonstration of our tuning method, we have constructed a laser based on a $3 \mathrm{~mm}$ long $5 \% \mathrm{Yb}: \mathrm{KY}\left(\mathrm{WO}_{4}\right)_{2}$ $(\mathrm{Yb}: \mathrm{KYW})$ crystal, cut for propagation along the b-axis. The pump laser was a diode bar at $980 \mathrm{~nm}$, that was focused through a flat laser input coupler mirror to a focal spot with radii $\left(1 / \mathrm{e}^{2}\right)$ of $100 \mu \mathrm{m} \times 80 \mu \mathrm{m}$ in the crystal. Wavelength locking was done by means of a reflective volume Bragg grating in a retroreflector design, as explained above. The retroreflector in this work was constituted by a volume Bragg grating together with a flat dielectric mirror, specified for HR between $1010 \mathrm{~nm}$ and $1050 \mathrm{~nm}$ for $63^{\circ}$ to $77^{\circ}$ angle of incidence, respectively. The Bragg grating had a peak reflectivity for plane waves at normal incidence of $97 \%$ at $1063.5 \mathrm{~nm}$ and a FWHM bandwidth of $0.55 \mathrm{~nm}$. The cavities we used are depicted in Fig. 1. The curved mirror imaged a small mode size of $\sim 80 \mu \mathrm{m}$ in the laser crystal in one arm onto a large mode size of $\sim 300 \mu \mathrm{m}$ on the Bragg grating in the other arm. We used a 200 $\mathrm{mm}$ radius of curvature mirror, with a total length of $122 \mathrm{~mm}$ in the first arm and $350 \mathrm{~mm}$ total length in the other arm. The retroreflector with the Bragg grating, which is described above, was placed in the far end of the long arm, and finally the cavity was completed with a flat mirror, $R_{1}$, as depicted in Fig. $1 \mathrm{~A}$. The reflectivity of $R_{1}$ was varied between HR and $85 \%$. For the HR case, the cavity has two output beams, denoted by $\mathrm{P}_{1}$ and $\mathrm{P}_{2}$, with one more added for the $85 \%$ case, denoted by $\mathrm{P}_{3}$. Another version of the Bragg grating arm of this cavity was also utilized. Here, the Bragg grating was used at normal incidence, as shown in Fig. 1B, which would lock the laser at the reflection peak for normal incidence at $1063.5 \mathrm{~nm}$. 


\section{CThE1.pdf}

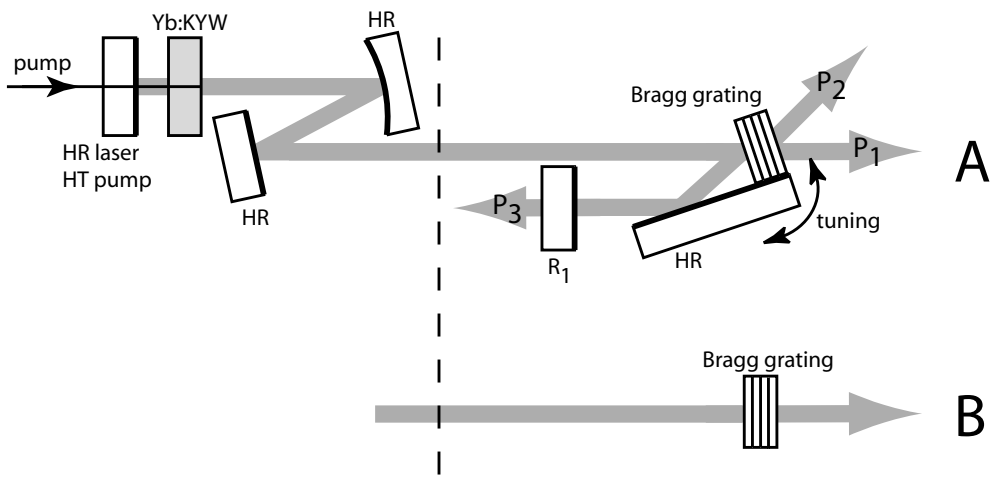

Fig. 1. The various cavity designs used.

The Bragg-locked laser with the retroreflector design, cavity A, showed a spectral bandwidth of $<0.1 \mathrm{~nm}$ FWHM at maximum pump power. The wavelength of the laser could easily be continuously tuned by rotating the retroreflector. The tuning interval ranged from $1000 \mathrm{~nm}$ to $1050 \mathrm{~nm}$ at maximum pump power, with $\mathrm{R}_{1}=\mathrm{HR}$, as illustrated in Fig. 2 . With an increased outcoupling for $\mathrm{R}_{1}=85 \%$, the upper bound dropped to $1043 \mathrm{~nm}$. In both cases the output power dropped sharply below $1000 \mathrm{~nm}$ due to decreased reflectivity of the input coupler.

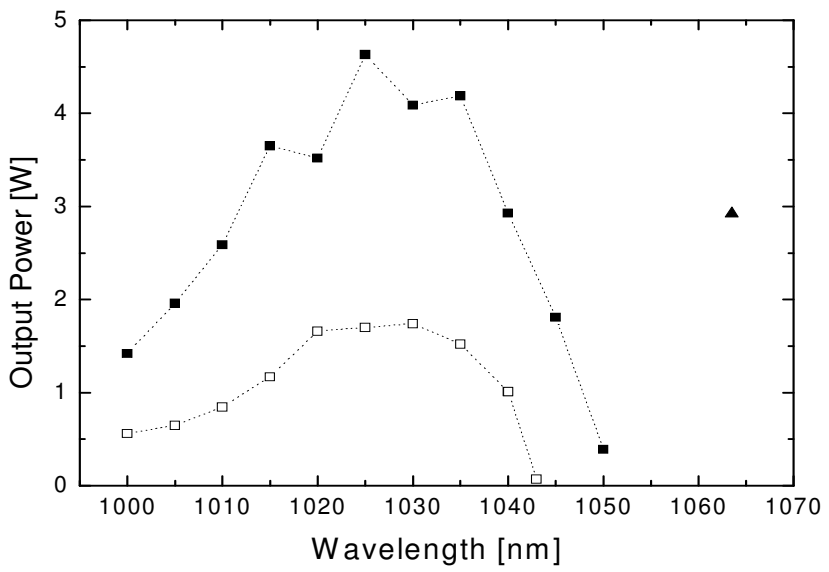

Fig. 2: Output power versus wavelength for cavity A with respect to total power (solid squares) and to $\mathrm{P}_{3}$ (empty squares). The output power of cavity B is shown with a solid triangle.

With the Bragg grating at normal incidence, cavity B, the laser was locked to a wavelength of $1063.7 \mathrm{~nm}$. The output power of $3 \mathrm{~W}$ shows that it would be possible to lock an $\mathrm{Yb}: \mathrm{KYW}$ laser to any fixed wavelength between at least $1000 \mathrm{~nm}$ and $1070 \mathrm{~nm}$. More specifically, all Nd ${ }^{3+}$ wavelengths for the ${ }^{4} \mathrm{~F}_{3 / 2} \rightarrow{ }^{4} \mathrm{I}_{11 / 2}$ transition can be reached.

While the beam quality of $\mathrm{P}_{1}$ and $\mathrm{P}_{2}$ was rather poor, both $\mathrm{P}_{3}\left(\mathrm{M}^{2}<1.3\right)$ and cavity $\mathrm{B}\left(\mathrm{M}^{2}<2\right)$ showed good beam qualities. By increasing the reflectivity of the Bragg grating, it should be possible to concentrate all the output power of cavity A into $\mathrm{P}_{3}$, thereby achieving a single output beam with good beam quality.

Future developments would include using the Bragg grating as input coupler, to replace the expensive dichroic mirror with an element with better wavelength selectivity. This could extend the tuning range to still lower wavelengths. It would also be interesting to perform intra-cavity frequency doubling of the locked laser.

\section{References}

[1] B. Jacobsson, V. Pasiskevicius, and F. Laurell, "Single-longitudinal-mode Nd-laser with a Bragg-grating Fabry-Perot cavity", Opt. Express 14, 9284 (2006)

[2] F. Havermeyer, W. Liu, C. Moser, D. Psaltis, and G. J. Steckman, "Volume holographic grating-based continuously tunable optical filter," Opt. Eng. 43, 2017-2021 (2004).

[3] B. Jacobsson, J.E. Hellström, V. Pasiskevicius, F. Laurell, "Transversal mode transformation in reflective volume Bragg gratings, theory and experiments," presented at Advanced Solid-State Photonics 2007 (Vancouver, Canada) 